\title{
ARTICLE
}

Received 6 Aug 2015 | Accepted 5 Jan 2016 | Published 25 Feb $2016 \quad$ DOl: 10.1038/ncomms10638 OPEN

\section{Drug design from the cryptic inhibitor envelope}

\author{
Chul-Jin Lee ${ }^{1, \star}$, Xiaofei Liang ${ }^{2, \star}$, Qinglin Wu ${ }^{1, \star}$, Javaria Najeeb ${ }^{1, \star}$, Jinshi Zhao ${ }^{1}$, Ramesh Gopalaswamy ${ }^{2}$, \\ Marie Titecat ${ }^{3}$, Florent Sebbane ${ }^{3}$, Nadine Lemaitre ${ }^{3}$, Eric J. Toone ${ }^{1,2} \&$ Pei Zhou ${ }^{1,2}$
}

Conformational dynamics plays an important role in enzyme catalysis, allosteric regulation of protein functions and assembly of macromolecular complexes. Despite these well-established roles, such information has yet to be exploited for drug design. Here we show by nuclear magnetic resonance spectroscopy that inhibitors of LpxC-an essential enzyme of the lipid A biosynthetic pathway in Gram-negative bacteria and a validated novel antibiotic targetaccess alternative, minor population states in solution in addition to the ligand conformation observed in crystal structures. These conformations collectively delineate an inhibitor envelope that is invisible to crystallography, but is dynamically accessible by small molecules in solution. Drug design exploiting such a hidden inhibitor envelope has led to the development of potent antibiotics with inhibition constants in the single-digit picomolar range. The principle of the cryptic inhibitor envelope approach may be broadly applicable to other lead optimization campaigns to yield improved therapeutics.

\footnotetext{
${ }^{1}$ Department of Biochemistry, Duke University Medical Center, Durham, North Carolina 27710, USA. ${ }^{2}$ Department of Chemistry, Duke University, Durham, North Carolina 27708, USA. ${ }^{3}$ Inserm, Univ. Lille, CHU Lille, Institut Pasteur de Lille, CNRS, U1019-UMR 8204-CIIL-Center for Infection and Immunity of Lille, F-59000 Lille, France. * These authors contributed equally to this work. Correspondence and requests for materials should be addressed to P.Z. (email: peizhou@biochem.duke.edu).
} 
T he availability of high-resolution crystal structures of protein-inhibitor complexes has revolutionized the drug development process, enabling structure-aided design of improved therapeutics based on visual inspection of receptorligand interactions. However, it is increasingly recognized that high-resolution structures of protein-inhibitor complexes do not necessarily enable a successful lead optimization campaign, as the static structural models often fail to capture the conformational flexibility of receptors or their bound inhibitors ${ }^{1,2}$. In contrast to the largely static view of protein structures provided by crystallography, the discovery of ring flipping events of buried aromatic residues of the basic pancreatic trypsin inhibitor by NMR (ref. 3) has heralded the widespread observation of molecular motions within macromolecules in solution. Conformational dynamics involving side-chain rearrangement, domain reorganization and binding-induced structural remodelling has been shown to play important roles in enzyme catalysis ${ }^{4-7}$, allosteric regulation ${ }^{8}$ and nucleic acid function ${ }^{9}$. Molecular recognition of small molecules likewise alters protein dynamics ${ }^{0}$. Despite the extensive demonstration of conformational dynamics of macromolecules in solution, the application of such information to drug development has remained an unmet challenge.

In this study, we used solution NMR to investigate the conformational states of small-molecule inhibitors bound to LpxC, an essential metalloamidase that catalyses the deacetylation of UDP-(3-O-acyl)- $N$-acetylglucosamine during the biosynthesis of lipid $\mathrm{A}$ in Gram-negative bacteria ${ }^{11,12}$. We show that these enzyme-bound inhibitors dynamically access alternative, minor conformations in solution in addition to the ligand state observed in the crystal structure. Furthermore, we demonstrate that these ligand conformational states collectively define a cryptic inhibitor envelope that can be exploited for optimization of lead compounds.

\section{Results}

A cryptic inhibitor envelope invisible in crystal structures. We chose Aquifex aeolicus LpxC (AaLpxC) in the lipid A biosynthetic pathway (Supplementary Fig. 1) for structural and dynamics investigation due to its exceptional thermostability, which has enabled both NMR measurements and crystallographic studies (for example, refs 13-16). Pseudomonas aeruginosa LpxC (PaLpxC) was exploited when co-crystal structures of the desired AaLpxC-inhibitor complexes could not be obtained. As a starting point, we investigated the conformations of CHIR-090 and LPC-011 bound to AaLpxC, two inhibitors that share the same threonyl-hydroxamate head group, but differ in their tail groups (Supplementary Fig. 1, Supplementary Table 1). CHIR-090 features a substituted biphenyl acetylene tail group that competes with the acyl chain of the LpxC substrate to occupy the hydrophobic substrate passage of the enzyme ${ }^{14}$. Replacing the tail group of CHIR-090 with a substituted biphenyl diacetylene group generated LPC-011 with improved antibiotic activity due to minimization of $\mathrm{vdW}$ clashes with the substrate-binding passage ${ }^{16,17}$. To provide a direct comparison with solution NMR investigations, we determined the crystal structure of AaLpxC in complex with LPC-011 (Fig. 1a, Supplementary Table 2). This structure reveals a single conformation of the threonyl-hydroxamate head group in the active site, with the threonyl $\mathrm{C} \gamma 2$ methyl group packing against an invariant phenylalanine residue (F180 in AaLpxC) and the O $\gamma 1$ hydroxyl group forming a hydrogen bond with the catalytically important lysine residue (K227 in AaLpxC). The threonyl side chain of the inhibitor features a trans configuration with a $\chi^{1}$ angle of $180^{\circ}$, a rotameric state that is less energetically favourable
(7\% population of all threonine side chains in proteins) compared with the alternative rotameric states of gauche- $\left(\chi^{1}=-60^{\circ}\right)$ and gauche $+\left(\chi^{1}=60^{\circ}\right)$ collectively accounting for $92 \%$ of the observed threonine side-chain conformations ${ }^{18}$. Since the observed ligand conformation in the crystal structure represents an unfavourable $\chi^{1}$ rotameric state of the threonyl head group, we investigated whether this group could access alternative ligand conformations in solution.

Database analysis of high-resolution protein structures has indicated that amino acid side-chains adopt specific rotameric conformations $^{18}$, and side-chain motions can be approximated as conformational hopping between rotameric states ${ }^{19}$. Such motions occur over a wide range of timescales, from ns movement of surface exposed residues to $\mu \mathrm{s}-\mathrm{ms}$ timescale ring flipping in protein cores. To determine rotameric populations of the ligand threonyl side chain over a wide timescale, we synthesized isotopically labelled CHIR-090 and LPC-011 and measured the scalar couplings ${ }^{3} J_{\mathrm{NC} \gamma 2}$ and ${ }^{3} J_{\mathrm{C}} \mathrm{C} \gamma 2$ that are dependent on the $\chi^{1}$ angle of the threonyl side chain (Supplementary Fig. 2 and Supplementary Table 3). Specifically, a large ${ }^{3} J_{\mathrm{NC} \gamma 2}$ value of $\sim 1.9 \mathrm{~Hz}$ is consistent with a trans ${ }^{N C \gamma 2}$ relationship between the amide nitrogen and the $\mathrm{C} \gamma 2$ methyl group of the threonyl head group, corresponding to a $\chi^{1}$ angle of $-60^{\circ}$ (gauche- $\chi^{1}$ ), whereas a small value of $\sim 0.2 \mathrm{~Hz}$ reflects a gauche $e^{N C \gamma 2}$ relationship (gauche $+{ }^{N C \gamma 2}$ or gauche- ${ }^{N C \gamma 2}$ ), corresponding to $\chi^{1}$ angles of $180^{\circ}\left(\right.$ trans $\chi^{1}$ ) or $60^{\circ}$ (gauche+ $\left.\chi^{1}\right)$, respectively. An intermediate value reflects a populationweighted average between the trans ${ }^{N C \gamma 2}$ and gauche ${ }^{N C \gamma 2}$ states $^{20}$. A similar relation is noted for the ${ }^{3} J_{\mathrm{C}^{\prime} \mathrm{C} \gamma 2}$ coupling ${ }^{20}$. Thus simultaneous measurements of the ${ }^{3} \mathrm{~J}_{\mathrm{NC} \gamma 2}$ and ${ }^{3} \mathrm{~J}_{\mathrm{C}} \mathrm{C} \gamma 2$ scalar couplings enable the determination of the populations of all three rotameric states of the threonyl side chain ${ }^{19}$. Measurements of LPC-011 yielded a ${ }^{3} J_{\mathrm{NC} \gamma 2}$ coupling of $0.58 \pm 0.05 \mathrm{~Hz}$ and a ${ }^{3} J_{\mathrm{C}^{\prime} \mathrm{C} \gamma 2}$ coupling of $0.77 \pm 0.04 \mathrm{~Hz}$, corresponding to a predominant trans $\chi^{1}$ configuration with a population of $0.65 \pm 0.03$ (Fig. $1 \mathrm{~b}$, Supplementary Table 3). Such an observation is consistent with the ligand conformation in the AaLpxC/LPC-011 crystal structure (Fig. 1a). However, the measurements also revealed that the threonyl side chain of LPC-011 can readily access alternative, minor conformational states with a population of $0.23 \pm 0.03$ for the gauche- $\chi^{1}$ conformation and a population of $0.12 \pm 0.01$ for the gauche $+\chi^{1}$ conformation (Fig. 1b). Measurements of CHIR090 yielded a similar result, with a ${ }^{3} J_{\mathrm{NC} \gamma 2}$ coupling of $0.45 \pm 0.07 \mathrm{~Hz}$ and a ${ }^{3} J_{\mathrm{C}^{\prime} \mathrm{C} \gamma 2}$ coupling of $0.67 \pm 0.04 \mathrm{~Hz}$, corresponding to populations of $0.77 \pm 0.04$ for the trans $\chi^{1}$ configuration, $0.14 \pm 0.04$ for the gauche- $\chi^{1}$ configuration and $0.09 \pm 0.01$ for the gauche $+\chi^{1}$ configuration (Fig. 1b, Supplementary Table 3). Modelling of the threonyl side chain in the second-most-populated gauche- $\chi^{1}$ state indicates that the C $\gamma 2$ methyl group would experience vdW interactions with the hydrophobic component of the K227 side chain with the $\mathrm{O} \gamma 1$ hydroxyl group oriented towards solvent, leaving a cavity against the F180 side chain of AaLpxC (Fig. 1c). Although the proteinligand interactions in the gauche- $\chi^{1}$ rotameric conformation are less favourable than those in the ground state of the trans $\chi^{1}$ rotamer, the lack of optimal interactions is partially compensated by the intrinsic free energy difference of the rotameric states of the threonyl side chain that favours the gauche- $\chi^{1}$ rotamer over the trans $\chi^{1}$ rotamer in the unbound ligand. Taken together, these solution measurement-derived rotamers collectively portray an inhibitor envelope that can accommodate three substitutions at the $C \beta$ position of the threonyl head group (Fig. 1c).

To test this prediction, we merged the two conformations of the threonyl head group and generated $C \beta$-di-methyl substituted compounds with the third $C \beta$-substitution containing either a hydroxyl group (LPC-037) or an amino group (LPC-040) 
a

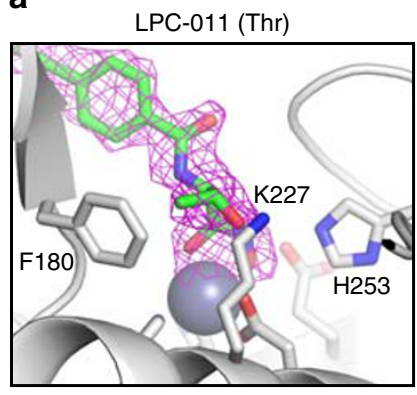

d

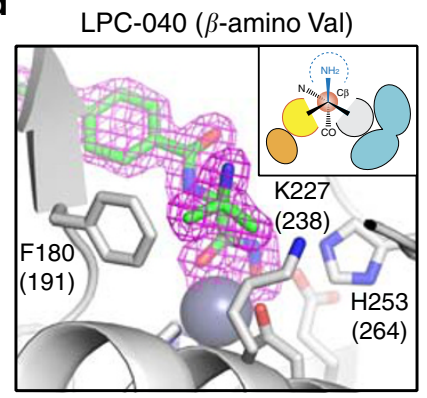

b

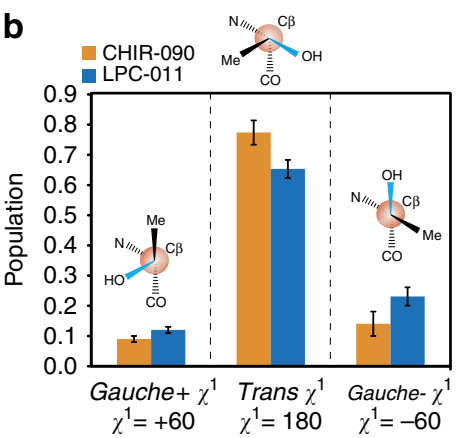

C

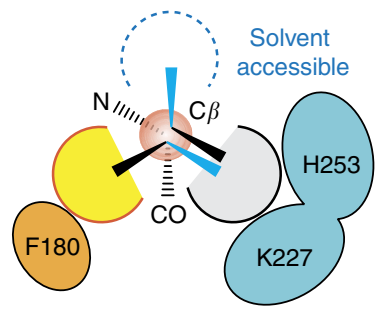

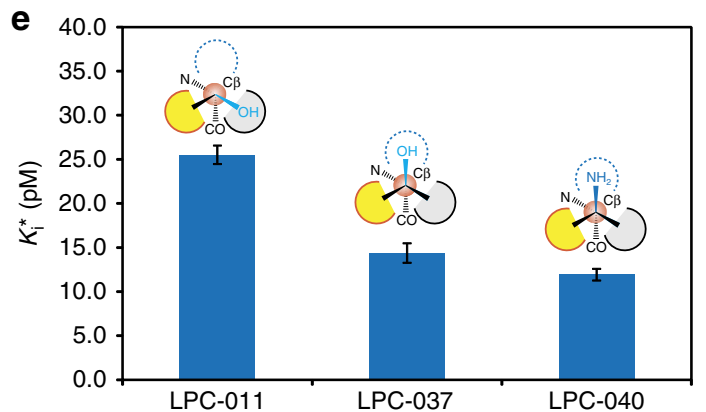

Figure 1 | Dynamic access of minor conformational states of LpxC inhibitors containing the threonyl head group. (a) Crystal structure of the AaLpxC/ LPC-011 complex, showing a single trans $\chi^{1}$ rotamer of the threonyl side chain of the inhibitor. AaLpxC is shown in the cartoon model and catalytically important residues in the stick model. LPC-011 is shown in the stick model, with the purple mesh representing the inhibitor omit map (2mFo-DFc) contoured at 1.0\%. (b) NMR measurements of scalar couplings $\left({ }^{3} J_{\mathrm{NC} \gamma 2}\right.$ and $\left.{ }^{3} J_{\mathrm{C}^{\prime} \mathrm{C} \gamma 2}\right)$ of the threonyl-head-group-containing LpxC inhibitors $\mathrm{CHIR-090}$ (orange) and LPC-011 (blue) reveal a dynamic distribution of all three rotameric $\chi^{1}$ states. (c) Combining the two most-populated ligand states creates a dynamically accessible inhibitor envelope around the $\mathrm{C} \beta$ atom of the threonyl head group. The binding pockets near F180 and H253/K227 are coloured in yellow and grey, respectively, and a third binding pocket accessible to solvent is denoted by an open dashed circle in blue. (d) The C $\beta$-triply substituted compound LPC-040 occupies all three pockets within the inhibitor envelope. PaLpxC is shown in the cartoon model, with catalytically important residues shown in the stick model. Residue numbering reflects the corresponding residues in AaLpxC, with PaLpxC residue numbers shown in parentheses. LPC-040 is shown in the stick model, with the purple mesh representing the inhibitor omit map ( $2 \mathrm{mFo}-\mathrm{DFc})$ contoured at $1.0 \sigma$. (e) Inhibition constants $\left(K_{\mathrm{i}}^{\star}\right)$ of $\mathrm{LpxC}$ inhibitors. Chemical substitutions at the C $\beta$-position of the inhibitors and their observed (LPC-011 and LPC-040) and predicted (LPC-037) binding modes within the inhibitor envelope are labelled.

(Supplementary Table 1). Structural analysis of LPC-040 in complex with PaLpxC indeed revealed the anticipated ligand conformation (Fig. 1d; Supplementary Table 2) with the two $\mathrm{C} \beta$-substituted methyl groups forming hydrophobic interactions with F180 (F191 $\left.{ }^{\text {PaLpxC}}\right)$ and the stem of K227 (K238 $\left.{ }^{\text {PaLpxC }}\right)$ and the amino group directed towards solvent accessible space to form a water-mediated hydrogen bond with the backbone carbonyl of F180 (F191 PaLpxC).

We next investigated whether these compounds show enhanced LpxC inhibition in enzymatic assays. Escherichia coli LpxC inhibition by CHIR-090 and LPC-011 both displayed slow-binding kinetics consistent with the transition from a rapid-forming initial encounter complex (enzyme-inhibitor complex (EI)) to the stable complex (EI*; Supplementary Fig. 3). Therefore, we focused enzymatic assays on the stable EI $^{*}$ complex. CHIR-090 and LPC-011 are potent LpxC inhibitors with $K_{\mathrm{i}}^{*}$ values of $153 \pm 8 \mathrm{pM}$ and $26 \pm 1 \mathrm{pM}$, respectively. Excitingly, the C $\beta$-triply substituted compounds LPC-037 and LPC-040 both showed enhanced LpxC inhibition, displaying $K_{\mathrm{i}}^{*}$ values of $14 \pm 1 \mathrm{pM}$ and $12 \pm 1 \mathrm{pM}$, respectively (Fig. 1e; Supplementary Table 4).

Drug design from the expanded inhibitor envelope. Having delineated the hidden inhibitor envelope at the $C \beta$ position of the threonyl head group, we next examined whether the dynamically accessible envelope of LpxC inhibitors can be further expanded at the $\gamma$ position. The molecule that fits this purpose is LPC-023 bearing an isoleucine-hydroxamate head group (Supplementary Table 1). Isoleucine shares a basic molecular scaffold with threonine, and its $\mathrm{C} \gamma 1-\mathrm{C} \delta 1$ group can be viewed as a substitution of the $\mathrm{O} \gamma 1$ group of threonine near the conserved lysine (K227 ${ }^{\text {AaLpxC; }}$ K238 ${ }^{\text {PaLpxC}}$ ) and histidine (H253 ${ }^{\text {AaLpxC; }}$ $\mathrm{H} 264^{\mathrm{PaLpxC}}$ ) residues. The isoleucine analogue was crystallized with AaLpxC (Supplementary Table 2), and two copies of the LpxC-inhibitor complexes were found in the asymmetric unit. Among the two protomers of LpxC, the second protomer (chain B) displayed a distorted active site with the catalytic H253 flipped out of the active site in a configuration that has not been observed in any of the previously reported LpxC structures. We reasoned that this would likely reflect a crystallization artifact and consequently focused our analysis on the first LpxC protomer (chain A) in complex with the isoleucine analogue, LPC-023 (Fig. 2a). In this protomer, the isoleucine head group displays a trans $\chi^{1}$ configuration, consistent with the predominant rotameric state observed in the threonyl group of CHIR-090 and LPC-011. The C $\delta 1$ methyl group adopts a gauche $+\chi^{2}$ conformation with regard to the $\mathrm{C} \alpha$ atom. In such a configuration, the $\mathrm{C} \delta 1$ methyl group is closest to and potentially forms $\mathrm{vdW}$ interactions with the nearby imidazole ring of the catalytic H253. This observation is somewhat surprising as the gauche+ $\chi^{2}$ angle is rarely observed for isoleucine in protein structures and contributes to $<5 \%$ of the observed $\chi^{2}$ rotamers, indicating that such a rotamer represents a high-energy state of the free ligand. 

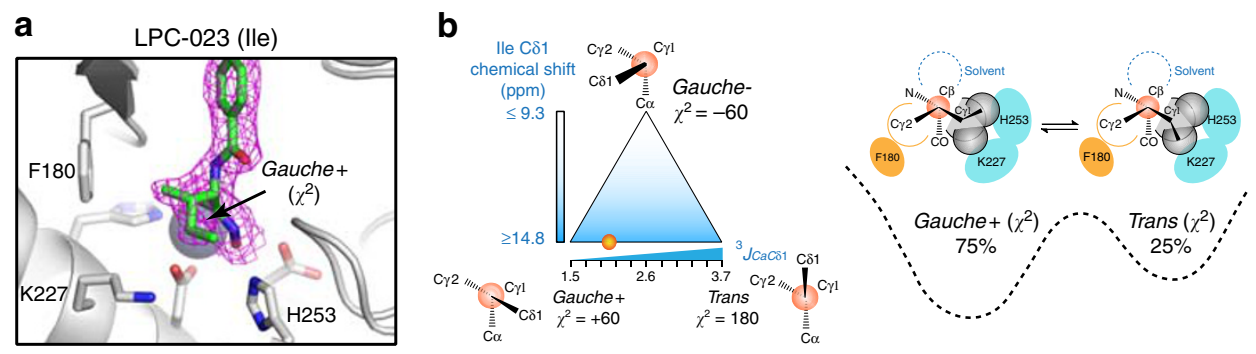

C

LPC-058

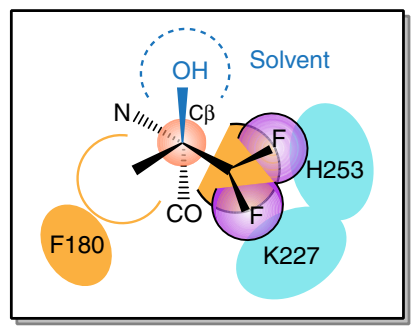

( $\beta$-difluoromethyl-L-allo-threonine)

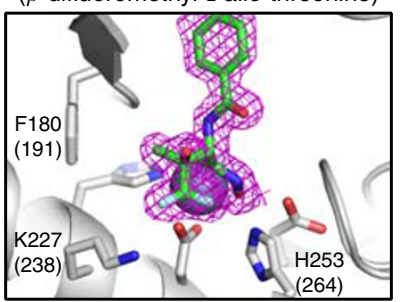

Figure 2 | Expanded inhibitor envelope enables the design of a potent inhibitor, LPC-058. (a) Crystal structure of AaLpxC in complex with LPC-023, an isoleucine derivative, reveals a gauche $+\chi^{2}$ rotamer conformation of the inhibitor. AaLpxC is shown in the cartoon model, with the catalytically important residues in the stick model. LPC-023 is shown in the stick model, with the purple mesh representing the inhibitor omit map ( $2 \mathrm{mFo-DFc)} \mathrm{contoured} \mathrm{at} 1.0 \sigma$. (b) Combined measurements of the $\mathrm{C} \delta 1$ chemical shift and the ${ }^{3} J_{C \alpha C \delta 1}$ coupling of LPC-023 in the protein-bound complex reveal a dynamic equilibrium between gauche + and trans $\chi^{2}$ rotameric states, with the gauche + state being the predominant conformation ( $\sim 75 \%$ population) and the trans state being the minor conformation ( $25 \%$ ). (c) Design and structural validation of LPC-058 that optimally occupies the inhibitor envelope. PaLpxC is shown in the cartoon model, with the catalytically important residues in the stick model. Residue numbering reflects the corresponding residues of AaLpxC, with PaLpxC numbers shown in parentheses. LPC-058 is shown in the stick model, with the purple mesh representing the inhibitor omit map (2mFo-DFc) contoured at $1.1 \sigma$.

We thus investigated whether the $\mathrm{C} \delta 1$ methyl group of the isoleucine analogue LPC-023 can access alternative $\chi^{2}$ rotameric states in solution using the isotopically labelled compound.

The isoleucine $\mathrm{C} \delta 1$ chemical shift is sensitive to its $\chi^{2}$ dihedral angle ${ }^{21}$. For the gauche + and trans $\chi^{2}$ rotamers, isoleucine $\mathrm{C} \delta 1$ methyl groups display downfield shifted chemical shifts of $>14.8$ p.p.m., whereas upfield shifted $\mathrm{C} \delta 1$ chemical shifts of $<9.3$ p.p.m. indicate a gauche- $\chi^{2}$ conformation. The unbound LPC-023 compound has a C $\delta 1$ chemical shift of 12.8 p.p.m. (Supplementary Fig. 4), consistent with rotameric averaging between a gauche- $\chi^{2}$ romateric state and the trans/gauche+ states. In contrast, the LpxC-bound LPC-023 displays a C $\delta 1$ chemical shift of 15.2 p.p.m. (Supplementary Fig. 4), indicating that the $\chi^{2}$ conformation resides entirely in the trans or gauche+ rotameric states or switches between these two states, but has no detectable population in the gauche- state (Fig. 2b, Supplementary Table 5). We next measured the ${ }^{3} J$ coupling between the $C \delta 1$ and $\mathrm{C} \alpha$ atoms (Supplementary Fig. 4). A trans configuration between $\mathrm{C} \alpha$ and $\mathrm{C} \delta 1$ would yield a large scalar coupling of $\sim 3.7 \mathrm{~Hz}$, whereas a gauche configuration would yield a small coupling of $\sim 1.5 \mathrm{~Hz}$ (ref. 21). Our measurements yielded a ${ }^{3} J_{\mathrm{C} \alpha \mathrm{C} \delta 1}$ coupling of $2.05 \pm 0.04 \mathrm{~Hz}$, corresponding to $75 \pm 2 \%$ population in the gauche $+\chi^{2}$ state with the $C \delta 1$ methyl group located adjacent to $\mathrm{H} 253$ and $25 \pm 2 \%$ population in the trans $\chi^{2}$ state with the same methyl group oriented towards K227 (Fig. 2b, Supplementary Table 5). Although the predominant gauche $+\chi^{2}$ rotameric state is consistent with the crystallographically observed inhibitor conformation, our NMR measurements support the notion that both the gauche + and trans states of the $\chi^{2}$ rotamers are conformationally populated and they collectively expand the inhibitor envelope at the $\gamma$-position, whereas the gauche- $\chi^{2}$ rotamer is energetically occluded and dynamically inaccessible in solution.
The delineation of two additional pockets that can accommodate methyl-sized functional groups to interact with side chains of the catalytically important histidine and lysine residues suggests fluorine as an attractive functional group for substitution. Fluorine has a slightly smaller size compared with the methyl group ${ }^{22}$, and the fluorine atom is both strongly electronegative and lipophilic ${ }^{23}$. This renders the fluorine group well-suited for forming hydrophobic interactions with the deprotonated histidine side chain and the stem of the lysine group, or forming electrostatic interactions with a protonated histidine imidazolium and a positively charged lysine terminal ammonium group.

Based on this analysis, we introduced difluoro substitution to the pro- $R$ methyl group of LPC-037 to yield LPC-058. Structural analysis of LPC-058 with PaLpxC indeed revealed the anticipated ligand conformation (Fig. 2c; Supplementary Table 2), with the $\beta$-methyl group occupying the hydrophobic pocket next to F180 (F191 $\left.{ }^{\text {PaLpxC }}\right)$ for vdW contacts, the $\beta$-hydroxyl group residing in the solvent pocket to form a water-mediated hydrogen bond with the backbone of F180 (F191 $\left.{ }^{\mathrm{PaLpxC}}\right)$, and finally with the difluoromethyl group oriented towards H253 (H264 PaLpxC) and $\mathrm{K} 227$ (K238 $\left.{ }^{\mathrm{PaLpxC}}\right)$. One of the fluorine atoms adopts a gauche + configuration with respect to $\mathrm{C} \alpha$ and forms a hydrogen bond with $\mathrm{N} \varepsilon 1$ atom of the protonated $\mathrm{H} 253\left(\mathrm{H} 264^{\mathrm{PaLpxC}}\right)$, while the second fluorine atom adopts a trans configuration with respect to $\mathrm{C} \alpha$ and forms an electrostatic interaction with the ammonium group of $\mathrm{K} 227$ (K238 $\mathrm{PaLpxC})$.

Excitingly, LPC-058 is an exceptionally potent inhibitor. It displayed slow-binding kinetics consistent with the rapid formation of an initial encounter complex (EI) followed by slow transition to the stable $\mathrm{EI}^{*}$ complex (Supplementary Fig. 5). Accordingly, $k_{\mathrm{obs}}$ increased hyperbolically over the inhibitor concentration $^{24}$. Steady-state kinetics analysis of the stable $\mathrm{EI}^{*}$ 

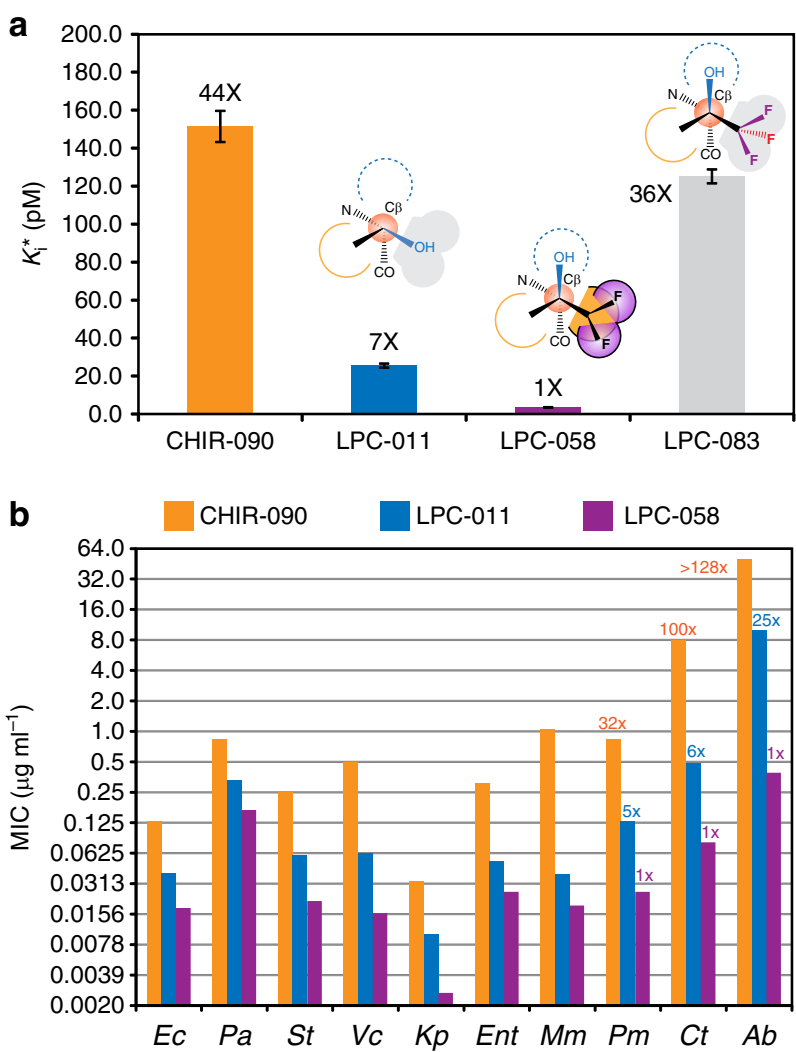

Figure 3 | LPC-058 is a superior inhibitor compared with the parent compounds LPC-011 and CHIR-090. (a) Inhibition constants of CHIR-090, LPC-011, LPC-058 and LPC-083. The head group of each compound and its conformation within the inhibitor envelope is denoted. (b) LPC-058 is a potent antibiotic and displays enhanced activity over LPC-011 and CHIR090 against a diverse array of Gram-negative pathogens. MIC enhancement of $>4$-fold over LPC-011 and $\geq 32$-fold over CHIR-090 is labelled. Tested bacterial species include E. coli $(\mathrm{Ec})$, P. aeruginosa $(\mathrm{Pa})$, Salmonella typhimurium (St), Vibrio cholerae (Vc), Klebsiella pneumoniae (Kp), Enterobacter cloacae (Ent), Morganella morganii (Mm), Proteus mirabilis (Pm), Chlamydia trachomatis ( $\mathrm{Ct}$ ) and Acinetobacter baumannii (Ab).

complex revealed an inhibition constant $\left(K_{\mathrm{i}}^{*}\right)$ of $3.5 \pm 0.2 \mathrm{pM}$, a 7-fold enhancement of potency over LPC-011 and a 44-fold enhancement over CHIR-090 (Fig. 3a). Incorporation of the $K_{\mathrm{i}}^{*}$ value into the analysis of the inhibitor concentrationdependent $k_{\text {obs }}$ values enabled accurate determination of the forward rate $\left(k_{5}=0.39 \pm 0.02 \mathrm{~min}^{-1}\right)$ and reverse rate $\left(k_{6}=0.0014 \pm 0.0001 \mathrm{~min}^{-1}\right)$ from EI to $\mathrm{EI}^{*}$ and the inhibition constant of the initial encounter complex EI $\left(K_{\mathrm{i}}=973 \pm 128 \mathrm{pM}\right)$.

To examine whether LPC-058 designed from the cryptic inhibitor envelope shows improved antibiotic activity over CHIR-090 and LPC-011, we determined its minimum inhibitory concentration (MIC) values against a range of Gram-negative pathogens. LPC-058 showed uniform improvement over CHIR090 and LPC-011 against all Gram-negative bacterial strains tested (Fig. 3b, Supplementary Table 6). In general, enhanced antibiotic activities of 2- to 4-fold over LPC-011 and 5- to 55-fold over CHIR-090 were observed for E. coli, P. aeruginosa, Salmonella typhimurium, Vibrio cholerae, Klebsiella pneumoniae, Enterobacter cloacae and Morganella morganii. More pronounced improvements (5- to 25-fold over LPC-011 and 32- to >128folds over CHIR-090) were observed for Proteus mirabilis, Chlamydia trachomatis and Acinetobacter baumannii. Of particular importance is the potent antibiotic activity of LPC-058 against Acinetobacter baumannii $\left(\mathrm{MIC}=0.39 \mu \mathrm{g} \mathrm{ml}^{-1}\right.$ ). To the best of our knowledge, LPC-058 is the first reported LpxC inhibitor with an MIC value below $1 \mu \mathrm{g} \mathrm{ml}^{-1}$ against this clinically important Gram-negative pathogen in vitro. The broadspectrum antibiotic activity of LPC-058 highlights the therapeutic potential of LpxC inhibitors as effective antibiotics against a wide range of Gram-negative infections.

\section{Discussion}

It is widely acknowledged that the dynamic interconversion of multiple conformational states is an intrinsic property of proteins and nucleic acids in solution. In comparison, the conformational dynamics of small molecules in their receptor-bound states has rarely been investigated, let alone utilized for drug design. Here we show that small-molecule inhibitors of LpxC dynamically access alternative, minor-state ligand conformations in addition to the predominant conformational state observed in crystal structures. These minor-state ligand conformations, together with that of the major state, collectively delineate a cryptic inhibitor envelope in solution that is invisible to crystallographic studies. Furthermore, we show that such a cryptic inhibitor envelope provides important molecular insights for the design of highaffinity ligands. In the case of LpxC inhibitors, analysis of the inhibitor envelope has led to the development of a potent antibiotic LPC-058. With inclusion of only three additional heavy atoms, the newly designed compound LPC-058 enhanced the inhibitory effect towards E. coli LpxC over its parent compound LPC-011 by 7 -fold and improved antibiotic activity by 2 - to 25-fold against a wide range of Gram-negative pathogens, rendering it the most potent and the most broad-spectrum LpxC inhibitor in vitro. Although some features of the LpxC inhibitor envelope, such as the solvent accessible pocket at the $C \beta$ position of the threonyl head group, might have been envisaged based on structural analysis of the LpxC/CHIR-090 complex ${ }^{14}$ and LpxC inhibitors bearing similar head groups to LPC-037 and LPC-040, but different tail groups, have been reported ${ }^{25,26}$, the precise definition of two accessory pockets at the $\mathrm{O} \gamma 1$-position of the threonyl head group could not have been predicted by structural analysis alone. In fact, the most widely employed functional substitution of a pro- $R$ methyl of LPC-037 is the trifluoromethyl group $\left(\mathrm{CF}_{3}\right)$, not the difluoromethyl group $\left(\mathrm{CF}_{2}\right)$ utilized in LPC-058. However, the trifluoromethyl substituted compound LPC-083 compromised the inhibitory effect $\left(K_{\mathrm{i}}^{*}=125 \pm 4 \mathrm{pM}\right)$ over its parent compound LPC-037 $\left(K_{\mathrm{i}}^{*}=14 \pm 1 \mathrm{pM}\right)$ by nearly ninefold (Supplementary Table 4 ). Its inhibition constant is worse than LPC-011 by fivefold (Fig. 3a), and it is a less potent antibiotic against $E$. coli $\left(\mathrm{MIC}=0.1 \mu \mathrm{g} \mathrm{ml}^{-1}\right.$ ) than LPC-011 $\left(\mathrm{MIC}=0.04 \mu \mathrm{g} \mathrm{m}^{-1}\right.$ ), which would have argued away from development of the synthetically more challenging $\beta$-difluoromethyl-allo-threonyl compound LPC-058 designed from the dynamic inhibitor envelope.

The work presented here departs from the established paradigm of ligand design from the crystallographically visible, static ligand conformation and highlights the potential of drug development from the 'invisible', dynamically accessible inhibitor envelope in solution, which encompasses the receptor-bound ligand conformations from both major and minor states. The framework presented here should be broadly applicable to lead optimization campaigns for small molecules, peptides and peptidomimetics to yield more effective therapeutics.

\section{Methods}

Chemical synthesis. Details of chemical synthesis and characterization are described in Supplementary Methods. 
Crystallography structural analysis. Protein samples of AaLpxC and PaLpxC were prepared as described previously ${ }^{16}$. Before crystallization trials, a fourfold molar excess of each compound, dissolved in DMSO, was mixed with $8 \mathrm{mg} \mathrm{ml}^{-1}$ AaLpxC (1-275, C181A) in $100 \mathrm{mM}$ potassium chloride, $2 \mathrm{mM}$ dithiothreitol and $25 \mathrm{mM}$ HEPES (pH 7.0) or $12 \mathrm{mg} \mathrm{ml}^{-1}$ PaLpxC (1-299, C40S) in $50 \mathrm{mM}$ sodium

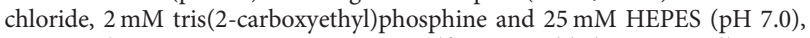
respectively. For PaLpxC, $10 \mathrm{mM}$ zinc sulfate was added as a crystallization additive. The protein-inhibitor mixture was incubated for $30 \mathrm{~min}$ at room temperature to obtain a homogenous sample. All of the LpxC-inhibitor complex crystals were obtained by the sitting-drop vapour diffusion method at $20^{\circ} \mathrm{C}$. Initial crystallization screening yielded microcrystals for the AaLpxC/LPC-011 complex in a reservoir solution containing 0.1 M HEPES ( $\mathrm{pH} 7.0$ ) and 15\% PEG 8000 and for the AaLpxC/LPC-023 complex in a reservoir solution containing $0.18 \mathrm{M}$ ammonium chloride, 11.8\% PEG3350 and 4\% 1,3-propanediol. The microcrystals were used to prepare seeding stocks by the Seed-Bead protocol (Hampton Research, HR2-320). Diffraction quality crystals were obtained by the streakseeding method. The final crystallization reservoirs contained $0.05 \mathrm{M}$ ammonium acetate and 10\% PEG3350 for the AaLpxC/LPC-011 complex and $0.18 \mathrm{M}$ ammonium chloride, 11.8\% PEG3350 and 10\% 1,3-propanediol for the AaLpxC/ LPC-023 complex, respectively. High quality crystals of the PaLpxC/LPC-040 and PaLpxC/LPC-058 complexes were obtained in precipitant solutions containing 0.1 $\mathrm{M}$ sodium acetate trihydrate $(\mathrm{pH} 4.8-5.1)$ and $2.4-2.6 \mathrm{M}$ ammonium nitrate. Crystals were cryoprotected using the corresponding mother liquor solutions containing 30\% 2-methyl-2,4-pentanediol (MPD) for the AaLpxC/LPC-011 complex, $30 \%$ ethylene glycol for the AaLpxC/LPC- 023 complex and 10\% glycerol for the PaLpxC/inhibitor complexes, respectively, before flash-freezing for data collection.

Data sets of the PaLpxC/LPC-040 and PaLpxC/LPC-058 complexes were collected in-house using a Rigaku MicroMax-007 HF rotating anode generator and R-Axis IV ++ detector. Data sets of the AaLpxC/LPC-011 and AaLpxC/LPC-023 were collected at the SER-CAT 22-ID beamline at the Advanced Photon Source at Argonne National Laboratory. The collected X-ray diffraction data were processed using HKL2000 (ref. 27) or XDS (ref. 28). The crystal structures of LpxC-inhibitor complexes were solved by molecular replacement with the programme PHASER (ref. 29) using PDB entries 3P3C and 3P3E for the AaLpxC-inhibitor complexes and the PaLpxC-inhibitor complexes, respectively. Restraints of the inhibitors were generated by using elBow (ref. 30) and edited manually. Iterative model building and refinement was carried out using COOT (ref. 31) and PHENIX (ref. 32). The $2 \mathrm{mFo}-\mathrm{DFc}$ omit maps ${ }^{33}$ were generated using PHENIX ${ }^{32}$.

Solution NMR measurements. Deuterated AaLpxC was expressed and purified as described previously ${ }^{13}$. The AaLpxC-inhibitor complexes were prepared by adding individual inhibitors to the purified protein in the presence of $5 \%$ deuterated dimethylsulfoxide (DMSO) in a 1:2 protein-inhibitor molar ratio, and incubated initially at room temperature and then at $45^{\circ} \mathrm{C}$ to form the complex. Samples were concentrated and exchanged into the NMR buffer containing $25 \mathrm{mM}$ sodium phosphate $\mathrm{pH} 7.0,100 \mathrm{mM} \mathrm{KCl}, 5 \%$ deuterated DMSO and $100 \% \mathrm{D}_{2} \mathrm{O}$. The NMR sample concentration was $\sim 1 \mathrm{mM}$.

The scalar couplings of ${ }^{3} \mathrm{~J}_{\mathrm{C}^{C} \gamma_{2}}$ and ${ }^{3} \mathrm{~J}_{\mathrm{NC} \gamma 2}$ for the AaLpxC/CHIR-090 and AaLpxC/LPC-011 complexes were measured on a Bruker $700 \mathrm{MHz}$ NMR spectrometer at $45^{\circ} \mathrm{C}$, using J-modulated ${ }^{1} \mathrm{H}_{-}{ }^{13} \mathrm{C}$ constant-time HSQC experiments $^{34,35}$. The reference and scalar coupling-modulated CT-HSQC spectra were recorded in an interleaved manner with a constant-time delay $(2 \mathrm{~T})$ set to $57.4 \mathrm{~ms}$, and the maximum evolution time for the indirect $\left({ }^{13} \mathrm{C}\right)$ dimension set $12.1 \mathrm{~ms}$. Data were processed using NMRPIPE (ref. 36) with eightfold zero-filling in the indirect dimension. The peak intensities were measured by SPARKY (ref. 37), and the ${ }^{3} J_{\mathrm{C} \mathrm{C}_{2} 2}$ and ${ }^{3} J_{\mathrm{NC} \gamma 2}$ couplings were calculated from the ratio of the peak intensities between the reference spectrum $\left(\mathrm{I}_{\text {ref }}\right)$ and the J-modulated spectrum $\left(\mathrm{I}_{\text {mod }}\right)$ according to equation (1):

$$
\frac{I_{\text {mod }}}{I_{\text {ref }}}=\cos (2 \pi J T)
$$

Rotameric populations were calculated based on the three-site jump model ${ }^{19}$ using values derived from self-consistent parameterization of ${ }^{3} \mathrm{~J}$ couplings ${ }^{20}$.

The scalar coupling ${ }^{3} J_{\mathrm{C} \alpha \mathrm{C} \delta 1}$ for the AaLpxC/LPC-023 complex was measured on an Agilent $800 \mathrm{MHz}$ NMR spectrometer at $37^{\circ} \mathrm{C}$ using a J-modulated constanttime ${ }^{13} \mathrm{C}$ HSQC experiment using selective Ile-C $\alpha$ inversion pulses. The ${ }^{3} J_{\mathrm{C} \alpha \mathrm{C} \delta 1}$ coupling was calculated from the ratio of the peak intensities between the reference spectrum $\left(\mathrm{I}_{\mathrm{ref}}\right)$ and the J-modulated spectrum $\left(\mathrm{I}_{\mathrm{mod}}\right)$ according to equation (1). Since the C $\delta 1$ chemical shift of 15.2 p.p.m. of LPC-023 excludes the gauche- $\chi^{2}$ rotamer ${ }^{21}$, populations of the remaining rotamers were calculated from ${ }^{3} J_{\mathrm{C} \alpha \mathrm{C} \delta 1}$ based on the two-site jump model between the gauche + and trans rotameric states $^{21}$.

Enzymatic assays. The radiolabelled substrate for the LpxC enzymatic assays, $[\alpha-$ $\left.{ }^{32} \mathrm{P}\right]$ UDP-3-O-[(R)-3-hydroxymyristoyl]- $N$-acetylglucosamine, and the unlabelled carrier substrate were prepared as previously described ${ }^{38}$. The assays were performed in a buffer consisting of $25 \mathrm{mM}$ HEPES pH 7.4, $100 \mathrm{mM} \mathrm{KCl}$, $1 \mathrm{mg} \mathrm{ml}^{-1} \mathrm{BSA}, 2 \mathrm{mM}$ dithiothreitol and $5 \mu \mathrm{M}$ substrate at $30^{\circ} \mathrm{C}$. Serial twofold dilutions of each inhibitor were prepared in DMSO and added to the reaction mixture with a 10 -fold dilution. The assays were initiated by addition of purified LpxC protein into the reaction mix with 1:4 dilution to the final concentration as specified.

The $K_{\mathrm{M}}$ value was determined by varying substrate concentrations from 0.4 to $50 \mu \mathrm{M}$ with $0.2 \mathrm{nM}$ of LpxC. To study the slow, tight-binding inhibition, LpxC activity was assessed in the presence of varying inhibitor concentrations. The product conversions were determined from $15 \mathrm{~s}$ up to $2 \mathrm{~h}$ after addition of $0.2 \mathrm{nM}$ enzyme for CHIR-090 and LPC-011 in the presence of $5 \mu \mathrm{M}$ substrate. Timedependent inhibition of LPC-058 was assayed in the presence of $30 \mu \mathrm{M}$ substrate and $0.1 \mathrm{nM}$ enzyme such that $k_{\text {obs }}$ can be extracted under the slow, but not tightbinding conditions ${ }^{39}$. The following time-dependent equation was used to fit the data:

$$
[P]=v_{s} t+\frac{v_{i}-v_{s}}{k_{o b s}}\left[1-e^{-k_{o b s} t}\right]+c
$$

with $v_{s}$ representing the steady-state rate, $v_{i}$ the initial rate, $k_{\mathrm{obs}}$ the rate of transition from the initial encounter complex to the final complex and $c$ the baseline.

The $K_{\mathrm{i}}^{*}$ was determined by analysing the rate of product accumulation after formation of the stable $\mathrm{EI}^{*}$ complex. $\mathrm{IC}_{50}$ curves for individual compounds were determined in the presence of $20 \mathrm{pM}$ of the enzyme and varying inhibitor concentrations. The Morrison's quadratic equation was used to fit the fractional activity data to determine $K_{\mathrm{i}}^{\star}$ app:

$$
\frac{v_{i}}{v_{0}}=1-\frac{[E]_{T}+[I]_{T}+K_{i}^{* a p p}-\sqrt{\left([E]_{T}+[I]_{T}+K_{i}^{* a p p}\right)^{2}-4[E]_{T}[I]_{T}}}{2[E]_{T}}
$$

where $[E]_{T}$ and $[\mathrm{I}]_{\mathrm{T}}$ represent the total enzyme and inhibitor concentrations, respectively. The inhibition constant $K_{\mathrm{i}}^{*}$ is converted from $K_{\mathrm{i}}^{\star}$ app according to the following relationship:

$$
K_{\mathrm{i}}^{*}=K_{i}^{* a p p} /\left(1+\frac{[S]}{K_{\mathrm{M}}}\right)
$$

For two-step slow-binding inhibition, kinetic parameters $k_{5}, k_{6}$ and $K_{\mathrm{i}}$ were extracted from curve fitting of experimental $k_{\text {obs }}$ values to inhibitor concentrations based on equations (5 and 6).

$$
k_{\mathrm{obs}}=k_{6}+\frac{k_{5}[I]}{K_{\mathrm{i}}^{\mathrm{app}}+[I]}=k_{6}+\frac{k_{5}[I]}{K_{\mathrm{i}}\left(1+[S] / K_{\mathrm{M}}\right)+[I]}
$$

$$
K_{\mathrm{i}}=K_{\mathrm{i}}^{*}\left(1+k_{5} / k_{6}\right)
$$

Measurements of the minimum inhibitory concentration (MIC). The MIC assay protocol was adapted from methods described in National Committee for Clinical Laboratory Standards (NCCLS) to using 96-well plates ${ }^{40}$. Bacteria were grown in the Mueller-Hinton medium at $37^{\circ} \mathrm{C}$ in the presence of varying concentrations of inhibitors and 5\% DMSO. To obtain more accurate readings of the MICs, three series of twofold dilutions of inhibitors were used. The starting concentrations of the three series are different by factors of 1.33 and 1.67, respectively. MICs were reported as the lowest compound concentration that inhibited bacterial growth.

\section{References}

1. Thanos, C. D., Randal, M. \& Wells, J. A. Potent small-molecule binding to a dynamic hot spot on IL-2. J. Am. Chem. Soc. 125, 15280-15281 (2003).

2. Carlson, H. A. \& McCammon, J. A. Accommodating protein flexibility in computational drug design. Mol. Pharmacol. 57, 213-218 (2000).

3. Wagner, G. \& Wuthrich, K. Dynamic model of globular protein conformations based on NMR studies in solution. Nature 275, 247-248 (1978).

4. Eisenmesser, E. Z. et al. Intrinsic dynamics of an enzyme underlies catalysis. Nature 438, 117-121 (2005).

5. Whittier, S. K., Hengge, A. C. \& Loria, J. P. Conformational motions regulate phosphoryl transfer in related protein tyrosine phosphatases. Science 341, 899-903 (2013).

6. Bhabha, G. et al. A dynamic knockout reveals that conformational fluctuations influence the chemical step of enzyme catalysis. Science 332, 234-238 (2011).

7. Boehr, D. D., McElheny, D., Dyson, H. J. \& Wright, P. E. The dynamic energy landscape of dihydrofolate reductase catalysis. Science 313, 1638-1642 (2006).

8. Tzeng, S. R. \& Kalodimos, C. G. Dynamic activation of an allosteric regulatory protein. Nature 462, 368-372 (2009).

9. Kimsey, I. J., Petzold, K., Sathyamoorthy, B., Stein, Z. W. \& Al-Hashimi, H. M. Visualizing transient Watson-Crick-like mispairs in DNA and RNA duplexes. Nature 519, 315-320 (2015).

10. Frederick, K. K., Marlow, M. S., Valentine, K. G. \& Wand, A. J. Conformational entropy in molecular recognition by proteins. Nature 448, 325-329 (2007).

11. Barb, A. W. \& Zhou, P. Mechanism and inhibition of LpxC: an essential zincdependent deacetylase of bacterial lipid A synthesis. Curr. Pharm. Biotechnol. 9, 9-15 (2008). 
12. Raetz, C. R. H. \& Whitfield, C. Lipopolysaccharide endotoxins. Annu. Rev. Biochem. 71, 635-700 (2002).

13. Coggins, B. E. et al. Structure of the LpxC deacetylase with a bound substrateanalog inhibitor. Nat. Struct. Biol. 10, 645-651 (2003).

14. Barb, A. W., Jiang, L., Raetz, C. R. \& Zhou, P. Structure of the deacetylase LpxC bound to the antibiotic CHIR-090: Time-dependent inhibition and specificity in ligand binding. Proc. Natl Acad. Sci. USA 104, 18433-18438 (2007).

15. Whittington, D. A., Rusche, K. M., Shin, H., Fierke, C. A. \& Christianson, D. W. Crystal structure of LpxC, a zinc-dependent deacetylase essential for endotoxin biosynthesis. Proc. Natl Acad. Sci. USA 100, 8146-8150 (2003).

16. Lee, C. J. et al. Species-specific and inhibitor-dependent conformations of LpxC: implications for antibiotic design. Chem. Biol. 18, 38-47 (2011).

17. Liang, X. et al. Syntheses, structures and antibiotic activities of LpxC inhibitors based on the diacetylene scaffold. Bioorg. Med. Chem. 19, 852-860 (2011).

18. Lovell, S. C., Word, J. M., Richardson, J. S. \& Richardson, D. C. The penultimate rotamer library. Proteins 40, 389-408 (2000).

19. Hennig, M. et al. Side-chain conformations in an unfolded protein: chil distributions in denatured hen lysozyme determined by heteronuclear ${ }^{13} \mathrm{C},{ }^{15} \mathrm{~N}$ NMR spectroscopy. J. Mol. Biol. 288, 705-723 (1999).

20. Perez, C., Lohr, F., Ruterjans, H. \& Schmidt, J. M. Self-consistent Karplus parametrization of ${ }^{3} \mathrm{~J}$ couplings depending on the polypeptide side-chain torsion chil. J. Am. Chem. Soc. 123, 7081-7093 (2001).

21. Hansen, D. F., Neudecker, P. \& Kay, L. E. Determination of isoleucine sidechain conformations in ground and excited states of proteins from chemical shifts. J. Am. Chem. Soc. 132, 7589-7591 (2010).

22. Meng, H., Clark, G. A. \& Kumar, K. in Fluorine in Medicinal Chemistry and Chemical Biology. (ed. Ojima, I.) 411-446 (Blackwell Publishing Ltd, 2009).

23. Wang, J. et al. Fluorine in pharmaceutical industry: fluorine-containing drugs introduced to the market in the last decade (2001-2011). Chem. Rev. 114, 2432-2506 (2014)

24. Vogt, A. D. \& Di Cera, E. Conformational selection or induced fit? A critical appraisal of the kinetic mechanism. Biochemistry 51, 5894-5902 (2012).

25. Mansoor, U. F. et al. Design and synthesis of potent Gram-negative specific LpxC inhibitors. Bioorg. Med. Chem. Lett. 21, 1155-1161 (2011).

26. Fei, Z. et al. A scalable synthesis of a hydroxamic acid LpxC inhibitor. Org. Process Res. Dev. 16, 1436-1441 (2012).

27. Otwinowski, Z. \& Minor, W. Processing of X-ray diffraction data collected in oscillation mode. Methods Enzymol. 276, 307-326 (1997).

28. Kabsch, W. XDS. Acta Crystallogr. D Biol. Crystallogr. 66, 125-132 (2010)

29. McCoy, A. J. et al. Phaser crystallographic software. J. Appl. Crystallogr. 40, 658-674 (2007)

30. Moriarty, N. W., Grosse-Kunstleve, R. W. \& Adams, P. D. electronic Ligand Builder and Optimization Workbench (eLBOW): a tool for ligand coordinate and restraint generation. Acta Crystallogr. D Biol. Crystallogr. 65, 1074-1080 (2009).

31. Emsley, P. \& Cowtan, K. Coot: model-building tools for molecular graphics. Acta Crystallogr. D Biol. Crystallogr. 60, 2126-2132 (2004).

32. Adams, P. D. et al. PHENIX: a comprehensive Python-based system for macromolecular structure solution. Acta Crystallogr. D Biol. Crystallogr. 66, 213-221 (2010).

33. Terwilliger, T. C. et al. Iterative-build OMIT maps: map improvement by iterative model building and refinement without model bias. Acta Crystallogr. D Biol. Crystallogr. 64, 515-524 (2008).

34. Grzesiek, S., Vuister, G. W. \& Bax, A. A simple and sensitive experiment for measurement of JCC couplings between backbone carbonyl and methyl carbons in isotopically enriched proteins. J. Biomol. NMR 3, 487-493 (1993).

35. Vuister, G. W., Wang, A. C. \& Bax, A. Measurement of three-bond nitrogencarbon $\mathrm{J}$ couplings in proteins uniformly enriched in ${ }^{15} \mathrm{~N}$ and ${ }^{13} \mathrm{C} . \mathrm{J} . \mathrm{Am}$. Chem. Soc. 115, 5334-5335 (1993).

36. Delaglio, F. et al. NMRPipe: a multidimensional spectral processing system based on UNIX pipes. J. Biomol. NMR 6, 277-293 (1995).
37. Goddard, T. D. \& Kneller, D. G. Sparky 3 (Univeristy of California, 2008).

38. Jackman, J. E., Raetz, C. R. H. \& Fierke, C. A. Site-directed mutagenesis of the bacterial metalloamidase UDP-(3-O-acyl)-N-acetylglucosamine deacetylase (LpxC). Identification of the zinc binding site. Biochemistry 40, 514-523 (2001).

39. Zhang, R. \& Windsor, W. T. In vitro kinetic profiling of hepatitis C virus NS3 protease inhibitors by progress curve analysis. Methods Mol. Biol. 1030, 59-79 (2013).

40. National Committee for Clinical Laboratory Standards. Approved Standard M7A1: Methods For Dilution Antimicrobial Susceptibility Test For Bacteria That Grow Aerobically. Clinical and Laboratory Standards Institute, Wayne, PA, USA (1997).

\section{Acknowledgements}

Diffraction data of LpxC-inhibitor complexes were collected at the Duke Macromolecular X-ray Crystallography Shared Resource and at the Southeast Regional Collaborative Access Team (SER-CAT) 22-ID beamline at the Advanced Photon Source, Argonne National Laboratory, supported by the US Department of Energy, Office of Science and the Office of Basic Energy Sciences under Contract number W-31-109-Eng-38. NMR data were collected at the Duke University NMR Center. This work was supported by National Institutes of Health grants AI055588 and AI094475, and the Duke Bridge Fund awarded to P.Z. The authors would like to acknowledge Drs Bidong D. Nguyen and Raphael Valdivia for providing the MCC (minimal chlamydiacidal concentration) measurements of LpxC inhibitors against Chlamydia trachomatis, and Dr Xin Chen for insightful discussions on compound synthesis.

\section{Author contributions}

P.Z. conceived the project. C.-J.L. and J.N. determined crystal structures of LpxCinhibitor complexes; X.L. and R.G. synthesized LpxC inhibitors under the direction of E.J.T.; Q.W. performed NMR studies; J.Z. carried out enzymatic assays; and J.Z., C.-J. L., M.T., F.S. and N.L. determined the antibiotic activities of LpxC inhibitors. P.Z. wrote the manuscript with critical inputs from all authors.

\section{Additional information}

Accession codes: The coordinates for the X-ray structures have been deposited to the Protein Data Bank (PDB) with accession codes of 5DRO, 5DRQ, 5DRP and 5DRR for the AaLpxC/LPC-011, PaLpxC/LPC-040, AaLpxC/LPC-023 and PaLpxC/LPC-058 complexes, respectively.

Supplementary Information accompanies this paper at http://www.nature.com/ naturecommunications

Competing financial interests: P.Z. and E.J.T. declare a competing financial interest. A patent on designed LpxC inhibitors was awarded to P.Z. and E.J.T. The remaining authors declare no competing financial interest.

Reprints and permission information is available online at http://npg.nature.com/ reprintsandpermissions/

How to cite this article: Lee, C.-J. et al. Drug design from the cryptic inhibitor envelope. Nat. Commun. 7:10638 doi: 10.1038/ncomms10638 (2016)

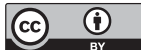

This work is licensed under a Creative Commons Attribution 4.0 International License. The images or other third party material in this article are included in the article's Creative Commons license, unless indicated otherwise in the credit line; if the material is not included under the Creative Commons license, users will need to obtain permission from the license holder to reproduce the material. To view a copy of this license, visit http://creativecommons.org/licenses/by/4.0/ 